Revue d'histoire de l'enfance « irrégulière »

Le Temps de l'histoire

$17 \mid 2015$

Naissance et mutation de la justice des mineurs

\title{
Émotion et opinion dans la justice des mineurs en France durant l'entre-deux-guerres
}

Emotion and opinion in the French juvenile system during the interwar period

Pascale Quincy-Lefebvre

\section{(2) OpenEdition}

Journals

Édition électronique

URL : http://journals.openedition.org/rhei/3848

DOI : $10.4000 /$ rhei.3848

ISSN : $1777-540 \mathrm{X}$

Éditeur

Presses universitaires de Rennes

Édition imprimée

Date de publication : 30 octobre 2015

Pagination : 149-167

ISBN : 978-2-7535-4215-0

ISSN : 1287-2431

Référence électronique

Pascale Quincy-Lefebvre, «Émotion et opinion dans la justice des mineurs en France durant l'entredeux-guerres », Revue d'histoire de l'enfance « irrégulière » [En ligne], 17 | 2015, mis en ligne le 30 octobre 2017, consulté le 05 mai 2019. URL : http://journals.openedition.org/rhei/3848 ; DOI : 10.4000/ rhei.3848 


\section{Émotion et opinion dans la justice des mineurs en France durant l'entre-deux-guerres}

Comment inscrire la réforme de la justice des mineurs dans le calendrier politique? $\mathrm{Au} x \mathrm{x}^{\mathrm{e}}$ siècle, alors qu'un espace public se démocratise, les politiques et les experts s'ouvrent davantage aux discours de l'émotion. La civilisation des médias est le théâtre de ces transformations. La France des années trente est propice aux engagements. L'enfance coupable est un levier et les « affaires » autour des « bagnes » d'enfants sont des moyens pour mobiliser l'opinion. En pleine période de lutte antifasciste, des hommes, des groupes dénoncent et interpellent. La grande presse joue sa partition. Les communistes donnent de la voix. L'indignation se focalise sur les établissements, l'impossible rééducation et la souffrance du mineur. Passion passagère ou mouvance des normes? L'émotion est fondatrice de communautés qui dépassent le cercle traditionnel des réformateurs de la justice des mineurs. De nouvelles représentations surgissent ou sont consolidées. À moyen terme, par les pressions et le jeu des mémoires, le processus de réforme se démocratise. La cause est efficace quand elle est en capacité de dépasser son objet: l'enfance coupable.

How did reform of the juvenile justice system find its way onto the political agenda? With the democratisation of the public sphere during the 20th century, both politicians and experts became more receptive to studies of emotions/emotional discourses. The burgeoning influence of the media provided the context for these transformations. During the 1930s, France was ready for change. Juvenile delinquency and the scandals regarding "children's prisons" became a public rallying point. At the height of the anti-fascist struggle, various individuals and groups condemned and challenged the juvenile justice system. The press played a major role in mobilising public opinion. The communists also lent their voice to the outcry. Public outrage focused on juvenile reform schools and the suffering produced by their inconceivable rehabilitation methods. Did this represent a shortlived obsession or a real transformation of existing standards? This emotional response brought together communities that went beyond the traditional juvenile justice reform groups. New representations emerged or were reinforced. As a result of public pressure and by playing on childhood nostalgia, the reform process underwent a medium-term transformation. The success of the cause was due to its ability to go beyond the focus on juvenile delinquency.

\author{
Pascale \\ QuincY-LefebvRe \\ Maître de confé- \\ rences en histoire à \\ l'université d'Angers. \\ Membre du Centre de \\ recherches historiques \\ de l'Ouest (CERHIO) \\ - UMR 6258.
}


1. Pour une approche historiographique, PlAMPER Jan, "L'histoire des émotions », traduit de l'anglais par Lisa Edmondson et révisé par Stéphane Péquignot, dans Granger Christophe, dir, À quoi pensent les historiens? Faire de l'histoire au XXI siècle, Paris, Autrement, 201, p. 225-240. Jan

Plamper a récemment publié GeschichteundGefühl. Grundlagen der Emotionsgeschichte (Histoire des sensibilités. Introduction à l'histoire des émotions), Siedler, 2012. Pour une réflexion critique sur les conséquences de l'irruption des émotions dans la pratique de l'histoire, Prochasson Christophe, L'Empire des émotions. Les historiens dans la mêlée, Paris, Demopolis, 2008.
2. Zelizer Viviana, Pricing the Priceless Child: The Changing Social Value of Children, Basic Books, 1985 (edition révisée en 1994) Princeton University Press.

3. Ambroise-Rendu AnneClaude et Delporte Christian (dir.), L'indignation. Histoire d'une émotion politique et morale, $X I x^{e}-x x^{e}$ siècles, Nouveau monde éditions coll. "Histoire culturelle" (CHCSC), 2008. Voir en particulier, YvoreL JeanJacques, « Les premières campagnes contre les bagnes d'enfants ", p. 105-128
Mots-clefs : enfance, justice des mineurs, médias, opinion publique, indignation

Keywords: childhood, juvenile justice, media, public opinion, indignation

T'histoire des émotions a le vent en poupe, non comme simple objet d'étude d'une discipline en " miettes ", mais comme possible paradigme des intrigues modernes et "catégorie d'analyse historique utile ${ }^{1}$ ». L'enfance est une réserve à sentiments pour une société médiatique en pleine expansion depuis la fin du XIX ${ }^{\mathrm{e}}$ siècle.

L'histoire de l'enfance, le développement d'une " économie morale de l'enfance " offrent un regard sur les modernités, leurs valeurs et normes ${ }^{2}$. Question plus pointue, l'attention portée à l'enfance dite coupable dans les médias est un indice de la fécondité des émotions dans le sentiment d'indignation et la nouvelle façon de penser l'action civile et politique. Parallèlement, la publicisation des "scandales" autour des "bagnes pour gosses " comme événement et processus éclaire le dynamisme des cultures de masse dans le changement social. Saisissons l'événement: avec quels acteurs et communautés, selon quelles utilisations pratiques et limites, l'émotion autour de l'enfant de justice travaille l'action?

Avec le développement d'une "culture du journal ", l'enfance malheureuse s'impose comme un réservoir d'indignation ${ }^{3}$. À partir de la fin du XIX ${ }^{e}$ siècle, puis avec une dimension nouvelle durant l'entre-deux-guerres, des faits divers deviennent des " affaires » et, par des " campagnes ", la société civile interpelle les politiques pour réformer le champ encore évanes- 
cent de la protection de l'enfance. La compassion des lecteurs s'appuie sur des représentations; elle suggère des histoires autour du sentiment d'enfance mais aussi dans le rapport aux risques sociaux et à la critique sociale. Les scandales des établissements pénitentiaires pour mineurs et leur exploitation par des campagnes d'opinion ont valeur d'indice sur la capacité à faire de l'enfant de justice une question sociale et politique ${ }^{4}$.

L'enfance dite coupable fut d'abord l'affaire d'un cercle restreint de philanthropes et experts. La question n'échappe pourtant pas à la démocratisation de la critique sociale. Avant 1914, Henri Rollet, avocat, homme d'œuvre, père de la grande loi de 1912 sur le tribunal pour enfants et adolescents, utilise les médias pour changer l'image des petits délinquants. Déjà l'émotion des masses est un argument dans le débat sur la loi de 1898 à propos des violences à enfant. Elle prend pour cible les mauvais parents 5 .

$\mathrm{Au}$ lendemain de la première guerre mondiale, les réformateurs placent leur espoir de changement dans l'application de la loi de 1912 sur les TEA. Auprès des masses, dans le cycle de l'attention publique, l'enfance coupable est une question marginale alors que les victimes sont nombreuses. Dans les années vingt, le journaliste Louis Roubaud publie Les enfants de Caïn dans Le Quotidien; il obtient son édition alors que le ton et le contenu irritent une Administration pénitentiaire qui avait collaboré. La polémique débouche sur des « réformes de papier ${ }^{6}$ ». Louis Roubaud, un ancien pupille de la paternelle de Mettray, passe à un autre sujet.

Durant les années trente, la dynamique évolue. Le sort des mineurs délinquants dans les établissements correctionnels est le ressort d'une indignation organisée. Non sans ambiguïté - Molière, en son temps, avec Tartuffe, avait dénoncé les "indignations calculées des grands prêtres de l'injustice ${ }^{7}$ " - mais non sans résultat. Dans une partie de l'opinion, l'enfant coupable devient victime. Pourquoi une telle accroche? Les faits d'opinion se nourrissent de l'actualité. Une campagne est une suite de dates et d'événements. Celle des «bagnes d'enfants » se caractérise par un temps fort: les années 1934-1937 et des pics correspondant à des " affaires ", Belle-Île en 1934 et Eysses en 1937. L'étude de la grande presse parisienne indique un essoufflement à partir de 1938. La concurrence est rude avec les nouvelles victimes dans un climat international tendu. Sur le terrain des mineurs de justice, une contre-offensive s'organise.
4. Capacité bien montrée dans « Les "bagnes d'enfants" en question. Campagnes médiatiques et institutions éducatives ", Revue d'histoire de l'enfance " irrégulière », n 13, 2011 , numéro dirigé par JeanJacques Yvorel auquel nous avons apporté une contribution.

5. Quelques années plus tard, la presse est à nouveau convoquée; des campagnes visent des établissements de réforme congréganistes ou proches des cléricaux et débouchent sur la fermeture du Bon pasteur de Nancy ou sur le procès de l'établissement congréganiste de Tours. À la même époque, le suicide d'un jeune bourgeois enfermé par sa famille à la "Maison paternelle » de Mettray défraie la chronique. L'institution est soutenue par le courant catholique. Le drame relance le débat sur le droit de correction des pères et sur le contrôle des œuvres privées.

6. Dupont-BOUChat MarieSylvie, PierRe Éric (dir.) avec Fecteau Jean-Marie, Trepanier Jean, Petit Jacques-Guy, SCHNAPPER Bernard, DekKER Jeroen, Enfance et justice au $x I x^{e}$ siècle. Essais d'histoire comparée de la protection de l'enfance 1820-1914. France, Belgique, Pays-Bas, Canada, Paris, PUF, 2001.

7. Citation empruntée à MATTEI Jean-François, L'Homme indigné. Essai, Paris, Cerf, 2012, p. 9. 
8. L'établissement de BelleÎle, plus connu sous son nom premier de « colonie agricole et maritime de Belle-Île-en Mer », a ouvert, sur décision ministérielle du 29 mai 1880, dans des locaux destinés, en 1848, à recevoir des prisonniers politiques. La création de colonies pénitentiaires privées est encouragée par la loi du 5 août 1850 portant sur l'éducation correctionnelle des mineurs délinquants.

9. FiLlaut Thierry (dir.), Une institution publique d'éducation surveillée. Belle île-en-Mer (1945-1977), CNFE-PJJ, 1996.
Le repli ne signifie pas l'oubli. Les campagnes ont un effet mémoire sur une génération d'acteurs aux avant-postes de la réforme après 1945.

\section{ETÉ 1934. Un CONTEXTE FAVORABLE}

\section{La révolte de Belle-Île}

Été 1934, des pupilles de la maison d'Éducation surveillée de Belle-Île se mutinent ${ }^{8}$. L'histoire est connue. Avec des professionnels du secteur, l'historien Thierry Fillaut a repris le dossier, croisant documents de presse et pièces administratives ${ }^{9}$. Les versions divergent; les journalistes ont produit des récits de l'instant. Lorsque l'information fait défaut, les "princes de la rédaction" comblent les manques car le souffle l'emporte sur l'exactitude.

Les rapports font état d'un incident dans le réfectoire. Il intervient dans un contexte chaud; des jeunes de la fanfare sont en conflit avec l'administration au sujet d'une non distribution de tabac. Au moment du repas, un surveillant sanctionne un manquement au règlement - les journalistes insisteront sur l'insignifiance de la faute - un colon aurait saisi son fromage avant que l'ordre n'en soit donné. Les coups pleuvent sur l'indiscipliné; des camarades cassent et s'en prennent physiquement aux adultes présents. Les rapports ne signaleront aucune blessure grave. Pour ne pas être punis, 55 mineurs s'évadent.

L'histoire bascule. En peu de temps, avec l'aide de quelques touristes et de la population locale, les mineurs sont rattrapés et violentés. La " mutinerie » n’est pas un événement extraordinaire. Les journalistes découvrent un fait divers. Le contexte fait surgir l'affaire.

Le lieu, la présence des touristes, la dimension collective de l'évasion: les ingrédients sont là pour une histoire. Quant au contexte, il favorise une exploitation sociale et politique. La publicisation est l'œuvre de professionnels de la presse. Elle résulte également de l'implication de militants communistes. Au fondement des récits et des actions, l'indignation est bien là. Elle a des sources humanistes communes. L'économie est différente même si des rencontres sont possibles.

\section{La grande presse sur les rangs}

À moindre coût, Belle-Île-en-Mer offre aux journalistes l'opportunité de jouer les Albert Londres. Le public est un habitué des bagnes, sujet d'indignation aux multiples rebondissements. La crise de la grande presse après la guerre a 
poussé les rédactions à mettre en avant l'action des reporteurs, leur rôle dans le dévoilement de ce qui serait caché, étouffé par les puissants. L'enfance coupable offre un exotisme de proximité et une figure de victime capable d'émouvoir sans trop diviser.

La grande presse parisienne est rapidement sur les lieux. L'impact de l'événement ne doit néanmoins pas être surestimé ou relu à la lumière de la légende qui devra beaucoup aux surréalistes en général et à Jacques Prévert en particulier. Dans un journal comme Paris-Soir, l'événement est couvert en deux temps. D'abord par un article isolé au moment des faits. Puis comme symbole d'une campagne lorsque le journaliste Alexis Danan, à son retour de vacances, saisit dans le moment, l'opportunité d'impliquer davantage son journal et sa personne dans la cause générale de l'enfance malheureuse et dans le sujet plus précis de la réforme de la justice des mineurs. Entré en 1926 à Paris-Soir alors que le titre était modeste, il doit s'adapter au nouveau format et aux ambitions du propriétaire, l'industriel Jean Prouvost.

Représentant d'une presse qualifiée de bourgeoise par des communistes actifs dans la campagne, interclassiste dans les faits, le journaliste développe une approche morale de l'événement. L'indignation doit rassembler plutôt que diviser. L'information a vocation à sensibiliser un lectorat appelé à voir dans le journaliste un acteur de la société démocratique et un appui pour les sans voix. À l'époque, Alexis Danan est une plume connue du milieu de la protection de l'enfance ${ }^{10}$ mais sa popularité récente et ciblée ne l'a pas encore habitué à être craint des administrations ou autres acteurs des politiques de l'enfance en danger ou coupable. Dans la nouvelle affaire, la partition du reporter est proche de celle de ses confrères: rallier le lectorat à une représentation principale: les jeunes sont victimes d'un système et d'un personnel que l'absence de réforme a figé dans des pratiques barbares et inefficaces socialement. Le discours expose publiquement la dignité bafouée des colons. Les mineurs ont été traités comme des bêtes. L'écrivain et journaliste occasionnel Roger Vaillant, auteur du premier $\operatorname{article}^{11}$ et d'autres parlent d'une « chasse à l'enfant ». Prévert en fera un poème. Cadeau fait aux journalistes en quête d'images fortes et évocatrices, l'expression est présente dans les premiers rapports du directeur à son administration pour désigner l'aide apportée par le public pour retrouver les fuyards ${ }^{12}$.

La presse parisienne dépeint le sort des jeunes détenus comme injuste: l'administration les traite comme des criminels alors que la justice les a acquit-
10. En particulier par la publication de Mauvaise graine, un ouvrage de 1931 qui reprend une enquête sur l'enfance en difficulté en stigmatisant l'inertie française tout en présentant au public les initiatives de réformateurs nationaux et étrangers, les apports des sciences médicales, psychologiques, du travail social et des pédagogies nouvelles.

11. Écrivain et dandy, Roger Vaillant a été embauché par Paris-Midi. Puis il écrit ponctuellement dans ParisSoir. Le plus souvent, il utilise un pseudonyme. Dans le cas de "La révolte des jeunes détenus. Des mutins de Belle-île, il ne reste plus qu'un seul évadé en liberté ", Paris-Soir, 30 août 1934, il signe « Robert François ".

12. A. D. du Morbihan, Y 475. Important dossier de presse à l'initiative du cabinet du préfet. Voir également A.N.- Fontainebleau, 19940500/227. Ministère de I'Intérieur, direction générale de la Sûreté générale (fonds " de Moscou "). Sous-dossier « Mutinerie à la Maison d'éducation surveillée de Belle-Île-enMer. Incident du 27 août $1934 »$ 
tés et soumis à une simple mesure d'éducation correctionnelle. Rapidement, les journalistes mettent en cause l'impossible réforme des établissements pour mineurs. Les brimades, la " chasse », les sanctions qui suivent l'évasion disent les failles d'un modèle qui, dans les établissements publics ou privés, recourt à un personnel incompétent car non formé aux fonctions de rééducation.

Passé à la commande, Alexis Danan revendique le fer. Dans une relation personnalisée avec son lectorat, il joue les chevaliers. Belle-Île est l'événement justifiant une plus vaste enquête pour appuyer une demande radicale de changement. Dans les semaines qui suivent, le journaliste ne lâche pas le sujet des établissements pour mineurs. L'investigation a évolué par rapport à Mauvaise graine, un ouvrage de 1931 reprenant une enquête sur "l'enfance anormale ". À l'époque, le journaliste veut convaincre par une enquête rigoureuse auprès des experts: il interroge et dénonce le retard de la France dans la prise en charge d'enfants susceptibles de rejoindre l'armée du crime ou la plèbe des assistés. Dans la campagne des bagnes d'enfants de l'automne 1934, le journaliste est l'homme du scandale, vrai croisé de la cause des mineurs. Devant l'inertie du pouvoir, il joue sur les émotions pour incarner et animer un mouvement de protestation populaire capable de peser sur les élus. Dans un champ concurrentiel (avec des quotidiens comme L'Euvre, des magazines comme $V u$ ), il capture des images sensibles. Les lecteurs des grands titres parisiens sont engagés dans un processus de modernisation du rapport à l'enfant davantage vu comme un semblable et un être faible à protéger. Les mêmes s'offusquent des insuffisances d'un État dont ils attendent davantage depuis la Grande Guerre. Le registre est celui de l'urgence dans un contexte troublé (crise économique, crise politique symbolisée par le 6 février 1934), propice aux engagements. L'investissement communiste est un exemple et une menace pour une cause, l'enfance malheureuse, traditionnellement défendue par des milieux plus réformistes. Laiguillon communiste radicalise les prises de parole et élargit le spectre des actions.

Entre grande presse et organisations militantes, les hommes, les mots circulent. Au début de la campagne, les identités sont marquées. Morale dans la grande presse, l'indignation est idéologique dans les organisations de la Troisième internationale. La stratégie du Front populaire à partir de la fin 34 ne fait pas disparaître les différences mais autorise une plus grande convergence au nom de l'intérêt de l'enfant, pour la démocratie et la justice. 


\section{Les organisations communistes à l'affût}

Les militants communistes sont les autres acteurs importants de la publicisation et de la fabrique de l'affaire de Belle-île. Le maillage du territoire est un atout. Dans les jours qui suivent, des conférences publiques sont organisées localement. Les associations satellites, le Secours rouge international - en qualité d'association de défense des détenus - le Secours ouvrier international (SOI) créé en 1924 « dans le vaste mouvement humanitaire qui accompagne la fin de la première guerre mondiale et dont le secours aux enfants constitue une des préoccupations essentielles $»-$ se mobilisent ${ }^{13}$. La présence communiste inquiète immédiatement les pouvoirs publics. Dès le 3 septembre 1934, la préfecture du Morbihan informe les services du ministère de l'Intérieur de la présence d'affiches apposées à Lorient le $1^{\mathrm{er}}$ septembre et annonçant une conférence publique dans cette ville, le 4 septembre, par le nommé " DIDIER », délégué du Secours rouge international, qui se propose de traiter $\mathrm{du}$ « bagne d'enfants de Belle Île et des récents incidents qui s'y sont produits ${ }^{14} »$.

Dans une étude bien documentée, Kari Evanson, américaine et spécialiste de littérature comparée, note des différences dans l'implication des deux collectifs puis davantage de convergences lorsque la ligne " front contre front " $s^{\prime}$ impose à tous ${ }^{15}$. Le Secours rouge agit d'abord en conformité avec la vulgate du parti sur la jeunesse prolétarienne. À la lumière de la crise économique et face au chômage, les organisations internationales communistes sont invitées à être attentives à toutes les injustices qui frappent cette dernière. Les révoltes préparent ou entretiennent l'idée de révolution. Les militants se doivent d'être attentifs au sort des mineurs de justice des établissements correctionnels, quintessence de la classe opprimée. Début septembre, le représentant du SRI, Didier, ancien métallo, enquête pour La Défense, le journal de l'organisation et publie un reportage «Avec les jeunes révoltés». Sur ses affiches, il appelle les Lorientais à venir écouter "La vérité " pour mieux se manifester car, précise le tribun: "Ces enfants vont être férocement punis si votre protestation n'arrête pas la main des bourreaux. " L'opinion est sommée de se manifester car, précise-til, "leur sort est entre vos mains ${ }^{16}$ ". La révolte est légitime et l'injustice doit cesser pour des jeunes exploités, maltraités par une administration au service du capital et des fascistes. Didier fait de l'indignation "vraie ", le levier d'une action qui doit rassembler sur une image de la jeunesse enfermée fort différente de celle que nombre de psychiatres diffusent à l'époque: des anormaux pour la
13. Association qui a vu le jour à Paris. À partir de décembre 1935, elle se « focalise exclusivement sur l'enfance malheureuse et devient l'Association nationale de soutien de l'enfance (ANSE) ». Evanson Kari,

«Vers le chemin de la vie: le discours communiste lors de la campagne médiatique contre les bagnes d'enfants, 1934-1938 ", Revue d'histoire de l'enfance «irrégulière », n 15, 2013, p. 190.

14. Joseph Clément Didier est membre du comité central de l'organisation. II est chargé d'une enquête à publier dans le journal du Secours rouge français, $\mathrm{La}$ Défense, ibid., p. 191.

15. Ibid., p. 187-204.

16. Cité par le commissaire central Nison de Lorient dans son rapport du $1^{\text {er }}$ septembre 1934 A. N. Fontainebleau, 19940500/227. Ministère de l'Intérieur, direction générale de la Sûreté générale (fonds « de Moscou »). Sous-dossier «Mutinerie à la Maison d'éducation surveillée de Belle-île-enMer. Incident du 27 août 1934 ». 
17. Birnbaum Pierre, Sur la corde raide. Parcours juifs entre exil et citoyenneté, Paris, Flammarion, 2002; ou autres ouvrages de l'auteur. grande majorité. La facture est classique mais décalée de la nouvelle ligne du parti de Maurice Thorez annoncée le 26 juin 1934. Le temps est désormais au front unique dans la lutte antifasciste.

Dans ce contexte, le Secours ouvrier international innove en devenant le canal d'une expression réformiste au service d'un projet politique de protection de l'enfance que symbolise l'action du médecin et psychologue Henri Wallon. Quelques semaines après Belle-Île, il signe la préface d'une brochure éditée par le SOI, Une plaie dans la société: les bagnes d'enfants qui dénonce mais également dessine un plan de réforme pour la rééducation de la jeunesse en danger.

Le camp communiste n'est pas univoque mais à l'été et l'automne 1934, le soupçon est la règle à l'égard des formes d'expression plus «bourgeoises » de la dénonciation. Le Secours rouge revendique une indignation « vraie » car " prolétarienne » et stigmatise l'action des réformateurs non communistes (experts, philanthropes), l'exploitation par la presse marchande. Ainsi Alexis Danan et Louis Rollin - homme politique plusieurs fois ministre, à la tête du groupe parlementaire pour la protection de l'enfance - sont publiquement suspectés de cléricalisme car Belle-Île est une maison d'Éducation surveillée laïque et publique. Rollin est un républicain du centre, catholique pratiquant. Danan est juif, agnostique, très proche de la SFIO. Ayant grandi en Algérie, le journaliste du grand capital appartient à ces " fous de République ${ }^{17}$ " qui ne transigent pas avec les principes.

L'affaire débouche sur une campagne. Le terrain est propice; la concurrence est un aiguillon pour l'action. La lutte anti-fasciste encourage la circulation des idées et favorise les projets des réformateurs. Parce que le sujet touche un large public, l'enfance est un trait d'union pour des coalitions de causes qui, au-delà du sujet, mobilisent sur le changement. Média de masse, Paris-Soir ne fait officiellement pas de politique. Hasard? Juste après Belle-Île, face au soupçon clérical, son journaliste, Alexis Danan, met à la une de son journal des scandales concernant les établissements d'inspiration catholique de Frasne et de Mettray.

Mettray, institution privée datant de la monarchie de Juillet, symbolise l'éducation correctionnelle. Son histoire, sa renommée, ses difficultés la placent dans l'orbite de la critique. Dans la foulée de l'affaire de Belle-île, Alexis Danan durcit le ton. Il produit un article au " picrate " où il accuse un surveillant chef à la retraite d'avoir tué des colons. Il songe à publier son texte dans $V u$, le magazine de Jean Vogel, proche du parti communiste et actif dans la campagne. Ne serait-ce pas là le moyen d'unir ceux qui attendent, de la révélation des 
scandales, une politique active de réforme? Dans L'Épée du scandale, un livre autobiographique de 1961, le grand reporter revient sur l'épisode. Il précise qu’à l'époque il ne pensait pas possible de sortir l'article dans Paris-Soir en raison des liens personnels unissant son patron, Jean Prouvost, à plusieurs grandes figures et familles catholiques soutenant Mettray. Pierre Lazareff l'aurait détrompé en obtenant l'autorisation du patron d'un titre en passe de devenir le journal le plus lu des Français. Sitôt l'article publié, la Paternelle, société gestionnaire de Mettray et le surveillant à la retraite (puis sa famille à son décès) engagent une action en justice qui ne débouchera sur un procès qu'en 1938.

Pour l'heure, en cet été et automne 1934, il y a bien deux campagnes et plusieurs discours. L'exemple de l'engagement communiste démontre la pluralité des positionnements et l'ancrage dans des enjeux politiques et sociaux qui surplombent la question de l'enfance en général, celle des enfants de justice en particulier. Après Belle-Île, la campagne contre « les bagnes d'enfants » fait écho à la dynamique frontiste de la lutte antifasciste. La protection de l'enfance et de la jeunesse est un thème pour des rassemblements. L'émotion est le langage pour convoquer le tribunal de l'opinion.

\section{4-1937, L'OPINION POUR DYNAMISER LES RÉFORMES}

Les établissements de réforme pour mineurs succèdent aux bagnes coloniaux dans les imaginaires de l'intolérable. La carte des émotions populaires auraitelle horreur du vide? L'investissement rencontre un sentiment d'enfance et des découvertes associées à l'adolescence et à la jeunesse. Entre 1934 et 1937 , le sujet est régulièrement à la une des grands médias. L'émotion est cyclique. Elle se nourrit de scandales révélés par la presse. Autour des " communautés émotionnelles ${ }^{18}$ ", des associations se créent pour dépasser des moments jugés trop fugaces ou les encadrer. La conviction est dans la force d'un mouvement d'opinion qui obligera les politiques à intégrer la question de la rééducation des mineurs de justice dans un calendrier de gouvernement.

\section{De coupable à victime : temporalités et mentalités}

La stratégie rencontre son public. Elle trouve à s'appuyer sur des représentations anciennes et nouvelles. Sur la longue durée, le thème de l'enfance malheureuse est bien représenté dans la littérature, dans les cultures scolaires
18. Le concept de " communauté émotionnelle » est emprunté aux travaux pionniers des années 1980 de Peter Stearns et Carol Zisowitz-Stearns. Théoriciens du phénomène qu'ils ont appelé emotionology, ils ont effectué une première tentative d'institutionnalisation avec la collection The History of Emotion, publiée par New York University Press. Une partie de la réflexion porte sur l'étude des phénomènes de communautarisation émotionnelle. Une attention est justement portée à la place prise par les " communautés textuelles" au sein desquelles les gens sont connectés par l'intermédiaire des médias. Travaux présentés par Plamper Jan, op. cit. 
19. Chauvaud Frédéric,

Justice et déviance à l'époque contemporaine. L'imaginaire, l'enquête et le scandale, Rennes, PUR, « Histoire », 2007.

20. Audoin-Rouzeau Stéphane, La Guerre des enfants (1914-1918), Paris,

A. Colin, 1993 et religieuses. À la suite de Michelle Perrot et parallèlement aux travaux de Dominique Kalifa sur les Bas-fonds, l'historien Frédéric Chauvaud relève la contribution du grand reportage et de la presse provinciale durant la Belle Époque. Les hauts faits des apaches, ces jeunes délinquants des fortifs, ont été mis en valeur par une presse prête à jouer des " paniques morales " pour vendre du papier. Ils n'ont pas chassé le Poulbot ${ }^{19}$.

Avant les années trente, rappelons que, sur le temps moyen, la guerre a été une école du sentiment et un moment pour l'irrationnel. Plus que d'autres conflits, 14-18 a sacralisé l'enfant. La culture de guerre le présente comme un enjeu justifiant le combat et une ressource pour l'idée de civilisation ${ }^{20}$. L'enfant en temps de paix offre l'opportunité de refaire société sur les victimes et de donner la parole à l'opinion, autre image de la Nation. La presse est vecteur et émetteur de nouvelles représentations qui accompagnent les espoirs et traumatismes d'une génération.

Sur le temps court et dans l'histoire immédiate, comme cela a été déjà observé, la campagne " contre les bagnes d'enfants " s'épanouit dans un contexte de crise économique, pourvoyeuse de malheurs sociaux pour les jeunes chômeurs des classes populaires. Face à la montée des périls, alors qu'ailleurs la jeunesse est embrigadée mais aussi exaltée comme force vitaliste, la sclérose des institutions renvoie la République à ses faiblesses. La fabrique de la loi est comme paralysée. Les études se multiplient pour dire l'incapacité à réformer et l'archaïsme des pratiques. Les modèles sont connus. La Belgique se fait légende pour la réforme. Psychiatres, psychologues, pénalistes, hommes et femmes d'œuvre, politiques s'épanchent sur la nécessité de rompre avec un modèle carcéral de rééducation des mineurs. Avec quel argent, sous quelle autorité? La mobilisation contre les « bagnes " d'enfants a valeur paradigmatique des espoirs et scléroses d'une démocratie qui doute d'elle-même ou du modèle à privilégier. L'enfant-victime est une valeur-refuge, un point de rassemblement. En plusieurs temps, la presse catalyse l'émotion et fait circuler les savoirs qui habituent la population à espérer dans les hommes de sciences en général et dans les médecins en particulier. Un mot nouveau surgit au cours de la campagne: "déficient ». Il est moins péjoratif qu’ " anormal ». Le journaliste Alexis Danan l'intègre dans le discours de presse. Dans un premier usage, encore peu habitué, il écrit " déficiant ». Novice, le correcteur laisse passer la coquille qui ne perturbe pas un public en pleine découverte. Le message passe: la caution scientifique qui, dans la déficience, transforme le coupable en victime. 
La déficience est médicale et sociale. Le traitement carcéral est scientifiquement inefficace, humainement mais aussi politiquement injuste. Ces nouvelles représentations fédèrent momentanément les partisans de la réforme. Henri Wallon apporte son soutien d'humaniste et d'homme de science. Il en appelle à la responsabilité des maîtres et à une éducation spécialisée capable d'évoluer. Auprès des populations, l'émotion parle plus fort. En 1936, des mouvements citoyens s'engagent à la relayer auprès du pouvoir.

\section{Fédérer des opinions}

Le fait divers est éphémère. La campagne inscrit le scandale dans un temps plus long mais le phénomène est perpétuellement menacé par le renouvellement de l'actualité. Les militants font preuve d'inventivité: dans les supports et les hommes à motiver. Presse, meeting, radio, cinéma: autant de médias qui parlent aux masses urbaines et se complètent avec un jeu d'emprunts noté par l'historien Jean-Jacques Yvorel ${ }^{21}$. L'association est le moyen d'organiser et de représenter une sensibilité qui se veut collective. Elle a une longue tradition dans le champ de l'enfance malheureuse. Dans la campagne des années trente, le phénomène prend les couleurs du temps. Les créations de l'année 36 sont, pour l'une inspirée par la contre-société communiste: des militants du SOI créent le "Comité de lutte contre les bagnes d'enfants", pour l'autre associée au pouvoir de la presse: le journaliste Alexis Danan crée avec le soutien de Louis Rollin, les comités de vigilance et de protection de l'enfance malheureuse.

Les deux associations entendent porter la voix d'une opinion indignée. De nature différente, comme la trajectoire de militants le démontre (certains passant de l'une à l'autre), elles sont susceptibles de dépasser les clivages idéologiques de départ pour afficher l'enfance comme cause humanitaire avec la charte de 1924 des droits de l'enfant comme référence ${ }^{22}$.

Le rapprochement bénéficie de la culture de la lutte antifasciste, de la place occupée par la défense des droits de l'homme et du citoyen en pleine période électorale. Lié à l'instant, il exploite des échanges plus anciens au cour de la nébuleuse réformatrice. Henri Wallon est le principal passeur entre experts et opinions d'une part et entre mouvements militants d'autre part ${ }^{23}$.

Les associations drainent un public hétérogène. Alexis Danan conçoit ses premiers comités de vigilance comme des extensions de la fonction d'interpellation d'un média de masse. Le siège de la Fédération se situe dans les locaux de
21. "Les liens que diverses productions culturelles entretiennent, les jeux d'emprunts, de recyclages qui produisent du sens pour le lecteur, le spectateur ou l'auditeur », YVOREL JeanJacques, «Présentation du dossier ", op. cit., Revue d'histoire de l'enfance "irrégulière ", n 13, 2011, p. 19.

22. Texte rédigé par Eglantyne Jebb de I'UISE. Les droits de l'enfant sont énoncés pour la première fois lors de la Déclaration des droits de l'enfant de Genève, en 1924, à l'initiative de l'influente association britannique, l'Union internationale de secours aux enfants (UISE), et concurremment à l'Association internationale de protection de l'enfance (AIPE) belgo-française. MARSHALL Dominique, « Dimensions transnationales et locales de l'histoire des droits des enfants. La Société des Nations et les cultures politiques canadiennes, 19101960 », Genèses 2/2008 $\left(n^{\circ} 71\right)$, p. 47-63.

23. Henri Wallon (18791962), connu pour ses travaux sur les stades de développement psychoaffectif de l'enfant. 
24. Van EtTen Henry, Chronique de la vie quaker française 1745-1945: deux siècles de vie religieuse, Paris, Société religieuse des Amis (Quakers), 1947. II est l'animateur de la revue, pôle du réformisme, Pour l'enfance coupable.
Paris-Soir. Le journaliste dispose du secrétariat du quotidien soucieux de créer une communauté de lecteurs et en quête d'un supplément d'âme à côté d'autres activités de prestige tournées vers le spectacle, le sport, l'international. La sociologie est celle du journal: mixte, importance des petites classes moyennes. L'éventail est plus large que le milieu traditionnellement investi dans les œuvres de protection de l'enfance en danger ou coupable. Le rassemblement se veut une réponse à l'indignation née du dévoilement de l'infortune des " petits bagnards » mais aussi des «petits martyrs » dans les familles que la société ne protège pas.

Le Comité de lutte contre les bagnes d'enfants est plus centralisé. Les quelques noms qui émergent montrent le projet de dépasser le cercle des militants des organisations communistes même si elles demeurent le pivot de l'initiative. Comme dans le mouvement d'Alexis Danan, quelques figures font penser aux résilients étudiés par Boris Cyrulnik, soucieux de témoigner et de faire cesser le scandale qui a anéanti leur jeunesse. Le Comité publie une revue intitulée Tribune de l'enfance. Il a à cœur l'organisation d'un grand congrès, acte symbolique et politique pour dynamiser la réforme et l'imposer comme un mouvement populaire en pleine période électorale.

\section{La nébuleuse réformatrice et le mouvement populaire}

Les réformateurs ont traditionnellement privilégié des réseaux de notables, des appuis politiques, une presse avertie. Des individualités (Henri Rollet ou Pauline Kergomard) d'avant 1914 avaient bien saisi l'intérêt des relations avec une opinion élargie. Après la première guerre mondiale, certains se plaignent de la difficulté à intéresser les médias de masse. Il faut revoir les méthodes mais tous n'ont pas les capacités, ni les convictions du quaker Henry Van Etten ${ }^{24}$. Lors de ses premières enquêtes, le journaliste Alexis Danan trouve des hommes et des femmes soucieux de faire entendre la voix de la réforme dans de nouvelles sphères. Le parlementarisme est en crise. L'interpellation des masses est le moyen de sortir de l'impasse législative et de l'inertie politique. Dans ce contexte, de grands experts comme Heuyer, Wallon mais aussi des politiques comme Louis Rollin reconnaissent une fonctionnalité à l'émotion. Leur caution, la dimension militante des écrits journalistiques et un Front populaire encourageant une démocratisation des expressions de la société civile favorisent une entrée en force de figures d'opinion dans le milieu de la protection de l'enfance. Les premiers 
concernés sont les journalistes. Les professionnels du reportage interviennent au titre de la "vérité » et comme représentants d'un quatrième pouvoir.

L'alliance entre des figures traditionnelles de la réforme et les acteurs d'une nouvelle médiatisation de la "cause de l'enfance " est particulièrement importante entre 1934 et 1937 . Une scène est caractéristique du rôle accru donné à la société civile dans la fabrique des politiques de l'enfance: l'animation d'une conférence publique par le député Louis Rollin et le reporter Alexis Danan à l'automne 1934. Le tandem répond à l'invitation du socialiste et polémiste Léo Poldés (1891-1970), fondateur du club du Faubourg, haut lieu du débat public parisien. Ce jour-là, le journaliste de Paris-Soir prend modèle sur Albert Londres; il fait monter à la tribune un colon, tout juste évadé de Frasne, un établissement de réforme dirigé par une congrégation religieuse. Devant la foule assemblée, il invite le mineur à décrire la " chime », une pratique disciplinaire particulièrement douloureuse et imposée aux pupilles. Dans un récit autobiographique, le journaliste décrit l'émotion partagée par la foule, le rôle que Rollin et lui-même lui attribuent dans le processus devant amener le changement:

L’enfant parut, à côté de moi, s'avança, justicier dérisoire, ébloui par les 《lumières du cintre, par les flashs des reporters qui s'étaient précipités. Il était livide et je me demandai s'il pourrait articuler son témoignage. Sa petite voix d'enfant, après les nôtres, pinçait les nerfs.

- Tout ce que vient de dire M. Alexis Danan est vrai d'un bout à l'autre, cria-t-il. Nous sommes battus, nous avons faim, oui, c'est vrai. Nous sommes malheureux comme des bêtes. La chime? Je vais vous dire ce que c'est.

Il se mit à mimer le supplice, étendant les bras, ouvrant les bras, fléchissant les jambes.

- Ici, une bûche, là, une autre bûche. Quand c'est trop lourd, quand un bras cède, le grand frère, derrière, c'est le moniteur, avec sa trique, et allez-y... Dix coups. Vingt coups. Sur le dos, sur la tête. Partout.

La salle haletait. Des voix de femmes suppliaient: “Assez! Assez!” Mais l'enfant était arrivé au bout de son effort.

Nous le portâmes dans les coulisses, à demi évanoui.

Rollin se leva. Il était à moitié cramoisi à éclater.

- Dès demain, cria-t-il, Alexis Danan et moi saisirons de ce scandale le ministre de la Justice. Faites-nous confiance. 
25. Danan Alexis, L'Épée du scandale. Trente ans au service des enfants perdus, Paris, Robert Laffont, 1961, p. 192.
Cette exhortation était nécessaire, car on percevait dans la salle des grondements d'émeute. La foule hurlait, trépignait, tendait le poing ${ }^{25}$.»

L'émotion est créée par la mise en spectacle de la parole et de la souffrance de la victime ainsi que par le mouvement de foule. Avec le sentiment, c'est un appel à l'action mais aussi le recours à un discours et à un processus de mobilisation basés sur l'urgence.

Dans une société d'information et d'opinion, la campagne est une mécanique rôdée. Le milieu de la protection de l'enfance ne se détourne pas d'une stratégie qui, associée aux formes plus habituelles d'action, trouve des relais dans la presse commerciale et auprès d'une population à l'éthos militant. Des journalistes entretiennent la culture de l'urgence par laquelle certains espèrent atteindre la réforme. Les professionnels vendent du papier; ils défendent un métier, une éthique. Ils acquièrent une meilleure place dans le jeu social. Bientôt, c'est comme experts et relais de l'opinion que certains siègent au Conseil Supérieur de Protection de l'Enfance. En octobre 1937, le reporter de Paris-Soir obtient cette reconnaissance. Le ministre Marc Rucart présente le Conseil comme un organe de liaison et de réflexion pour des réformes qui ont trop tardé. Il impose la présence de Louis Roubaud et d'Alexis Danan, ce dernier comme " rapporteur de la section de l'Enfance malheureuse ".

Ancien journaliste, Marc Rucart est successivement ministre de la Justice, puis de la Santé sous le Front populaire. Quelques mois plus tôt, il occupe la première fonction alors qu'un nouveau scandale relance le cycle compassionnel autour de l'enfant de justice. L'affaire Abel, du nom du colon décédé dans des conditions rapidement décrites comme non tolérables, intervient alors que la formation de moniteurs-éducateurs et leur envoi dans un établissement pilote de l'Éducation surveillée est un quasi fiasco. Manque de moyens? Manque de volonté politique? La mort du pupille de la colonie correctionnelle d'Eysses (Lot-et-Garonne) fait l'objet d'une large couverture médiatique dans la presse populaire et d'une offensive des organisations communistes. Nouveau pic, l'affaire est aussi un tournant et démontre les limites de la compassion d'une opinion tiraillée entre émotions et sécurité. 


\section{UNE ÉMOTION MOINS FONCTIONNELLE}

\section{APRÈS 37}

\section{L'affaire Abel, pic ou tournant?}

Le colon Abel décède d'une phtisie ou tuberculose galopante. La gravité de son état est tardivement diagnostiquée. Le jeune homme a dix-neuf ans. Il n’a commis aucun délit grave. Non condamné, après des passages en établissement de réforme sur demande des parents, puis en maison d'Éducation surveillée, il est interné à Eysses, une institution publique pour les condamnés et les indisciplinés. À son arrivée, il est mis en observation, c'est-à-dire à l'isolement plusieurs mois durant. Il quittera ce quartier pour rejoindre l'infirmerie, puis l'hôpital de Villeneuve ${ }^{26}$.

Le Secours rouge est une des premières organisations à rendre publique l'affaire. De jeunes communistes recueillent les dernières paroles $\mathrm{du}$ mourant et publient des courriers accusateurs du père dans la presse militante. La nouvelle du décès est suivie d'une vaste enquête. Marc Rucart se déplace et organise une conférence de presse devant un aréopage de journalistes. Les lecteurs de Paris-Soir, photos à l'appui, participent à l'aventure contée par le reporter Alexis Danan. Non sans tension avec des collègues présents sur les lieux, le journaliste se décrit tel un chevalier qui défend l'orphelin, guide le ministre pour lui révéler les lieux secrets du mal: en particulier le sinistre cachot $n^{\circ} 19$ suintant l'urine. L'émotion est garantie. Dans d'autres médias, des critiques se font plus nombreuses sur la campagne et ses méthodes. L'affaire réactive la compassion, une composante de l'esprit de réforme. Parallèlement,
26. Sur l'affaire «Abel », voir notre ouvrage, QuincYLefebvre Pascale, Combats pour l'enfance. Itinéraire d'un faiseur d'opinion: Alexis Danan (1890-1979), Paris, Beauchesne, 2014. 
27. Dans les années trente, le cinéma est devenu un des grands loisirs des classes populaires urbaines. Le passage au parlant date des années vingt et de nombreuses salles de quartiers sont équipées. Les dialogues du film sont d'Henri Jeanson. Les acteurs ont été choisis parmi des comédiens connus, Ginette Leclerc,

Annie Ducaux, ou débutants à l'exemple de Corinne

Luchaire. Le film de Moguy relève d'un genre établi et que d'autres œuvres viendront compléter aprèsguerre. Voir LE PAJOLEC

Sébastien, « La maison de correction dans le cinéma français. Apogée et disparition d'un stéréotype (milieu des années 1930-début des années 1960) », dans

BARdet J.-P., Luc J.-N., RoBinRomero I., Rollet C. (dir.), Lorsque l'enfant grandit. Entre dépendance et autonomie, Paris, Presses de l'université Paris-

Sorbonne, 2003, p. 817-828.

Le film est une production commerciale. D'autres sont de véritables outils de propagande à l'exemple du film soviétique de 1931, Le Chemin de la vie, s'inspirant librement de l'histoire de la « commune pédagogique » de Bolchevo (Ukraine) exploité en 1934 par le SOI. Voir CAROLI Dorena, L'Enfance abandonnée et délinquante dans la Russie soviétique (1917-1937)

Paris, L'Harmattan, 2004

Pour ce film, Nikolaï Ekk, remporte le prix du meilleur réalisateur à l'occasion de la Mostra de Venise en 1932.

28. Une nouvelle directrice est mandatée par le ministre de la Justice pour introduire de « nouvelles méthodes d'éducation ». Là où l'ancienne direction voyait des « garces », « des filles perdues » qui « ne cèdent que sous les coups » et dont " il faut se faire craindre ». La jeune femme entend réformer par la douceur et la confiance « des pauvres gosses sans défense ». Elle prend sous son aile Nelly, une jeune fille abusée par son beau-père. des critiques montent. Une contre-offensive s'organise. La même tension s'exprime à propos d'autres expressions de la campagne. En témoigne l'accueil de Prison sans barreaux, un long métrage du réalisateur Léonide Moguy projeté sur les écrans en février $1938^{27}$. L'œuvre est de fiction mais a pour "conseiller technique " Alexis Danan. L'histoire porte sur la réforme d'une maison pénitentiaire pour jeunes filles; le tout sur fond d'intrigue amoureuse $^{28}$.

\section{À partir de 1937, contre-campagne et désengagement}

Les campagnes de presse contre "les bagnes d'enfants " n'ont jamais occupé l'ensemble du terrain de la question des établissements pour mineurs dans les médias. Par exemple, en 1934, le lecteur accède à des discours critiques, hostiles dans une partie de la presse de province ou catholique. Le contexte de 1935 et de 1936 est plus favorable au consensus. Les critiques ressortent de façon structurée, à partir de 1937, dans le cadre de l'affaire Abel.

La contre-campagne a comme point d'orgue les "dérives» médiatiques. Un corpus serait à établir. Elle s'épanouit dans les milieux hostiles au Front populaire et alors que celui-ci se délite, elle atteint la presse commerciale. Les journalistes ont à leur disposition les arguments des agents de l'Administration pénitentiaire. Certains sont encouragés par les « amis » des associations gestionnaires d'établissements privés mis en cause dans la campagne. Les plus actifs cherchent à défendre l'œuvre de Mettray, le célèbre établissement devant fermer ses portes en 1937. La contre-campagne privilégie les " mensonges " sur les « victimes » et la nature des 
institutions. Le Matin se plaît à souligner que la moyenne d'âge à Eysses est de 19 ans $1 / 2$ et que l'établissement reçoit la lie de la population des mineurs de justice. Des journalistes mettent en cause les enquêtes à charge contre un personnel éprouvé. L'affaire Abel a débouché sur le renvoi du médecin de l'établissement, André Guy. Disposant d'une clientèle privée à Villeneuve-sur-Lot, le professionnel de santé exerce ses fonctions dans l'établissement depuis plus de trente-cinq ans. Décoré par l'Administration pénitentiaire, il a reçu la Légion d'honneur en 1931. Personnalité locale, il est le président du Syndicat médical du Lot-et-Garonne. Avec le soutien de ses confrères, se jugeant victime, l'homme diffuse brochures et tient meeting ${ }^{29}$.

Presse et acteurs institutionnels accusent les principaux animateurs de la campagne « contre les bagnes d'enfants » d'instrumentaliser la sensibilité des masses à des fins idéologiques ou marchandes et de déformer les faits. La tonalité de la critique du film Prison sans barreaux dans Ciné-France, Le journal des spectateurs, est sans équivoque. Sa nature indique un retournement de l'opinion:

Ce que nous reprochons au thème de Prison 《sans barreaux, c'est son "esprit" - très dangereux à notre avis - c'est celui d'une certaine presse dont Paris-Soir est le type même, qui cherche à nous apitoyer sur le sort des criminels, que l'on voudrait nous faire prendre pour des martyrs et des victimes de la société. On nous donne d'émouvants cas particuliers, comme celui de Nelly, puis on nous demande de généraliser. Ceci est trop facile! Les détenues qui vivent dans les maisons de redressement ne sont pas toutes des anges $^{30}$ !"
29. A. D. du Lot-et-Garonne, 1Y 95. Syndicat médical du Lot-et-Garonne, Le scandale d'Eysses près Villeneuvesur-Lot (d'après les documents officiels. La colonie correctionnelle d'Eysses, le scandale. Le problème de l'enfance délinquante), Villeneuve-sur-Lot, impr. Bador, 1937.

30. P. A., « Prison sans barreaux de Léonide Moguy », Ciné-France. Le Journal des spectateurs, $n^{\circ} 31,25$ février 1938 p. 6-7. La société doit aider les « brebis égarées » mais le chroniqueur juge simpliste et caricaturale, par exemple, l'opposition privé/public. Plus globalement il appelle le spectateur à se méfier de l'« esprit » dans lequel le sujet est monté. Cela ne l'empêche pas de rendre hommage à l'excellente réalisation et aux qualités techniques; Moguy a réussi un « très beau, un très émouvant film français ».
Le critique salue tout spécialement la « remarquable interprétation » et retient la révélation d'une jeune actrice de 17 ans, Corinne Luchaire, qui dans le rôle de Nelly est « extraordinaire de jeunesse, de sincérité ".Dans Pour l'enfance coupable d'Henry Van Etten, Claire Lyon note quelques invraisemblances. Plus intéressant au regard de la ligne défendue par les animateurs de la revue, l'auteure de la critique reproche au film d'insinuer que la maison de rééducation fonctionne mal tant qu'elle est entre les mains privées et se transforme lorsqu'elle est rachetée par l'État. La conclusion est plus positive : l'œuvre est jugée comme devant intéresser le public et, à ce titre elle « aidera beaucoup à la diffusion des idées de rééducation que nous souhaitons appliquer». Pour l'enfance coupable, $\mathrm{n}^{\circ} 22$, janvier-février 38, p. 14 
31. La grogne du Syndicat des agents de la Pénitentiaire s'exprime dans Le réveil pénitentiaire. Organe corporatif du syndicat national du personnel pénitentiaire de France et des colonies: la presse est accusée d'exciter les esprits des jeunes; d'influencer le gouvernement. En 1939, des auteurs du réveil réclament « justice ", ils mettent en cause « l'insécurité dans les MES », conséquence de «l'absence de répression » et de « l'humiliation du personnel ».

32. Jean Bancal s'en prend violemment aux journalistes qui, dans les années trente, et plus particulièrement en 1936, auraient fait beaucoup de mal au travail de réforme de l'Administration pénitentiaire. II mentionne des « campagnes de presse venimeuses, animées par des journalistes en mal de titres flamboyants, de reportages sensationnels et d'échos scandaleux, qui ont trouvé dans ce domaine une occasion d'émouvoir la sensibilité du public, de se poser en défenseurs d'enfants martyrs et faire de la publicité pour leur journal ». Jean Bancal conclut sa diatribe par l'expression de son « mépris » à l'égard de ces agitateurs qui auraient agi dans un esprit (celui de 1936) qu'il importe de combattre pour mener

autrement la réforme car de « telles questions ne se traitent pas sur le forum ".

Essai sur le redressement de l'enfance coupable.

Paris, Librairie du recueil Sirey, 1941, 77 p. Préambule, p. 8-9.

33. BoltanSKI LUC, La Souffrance à distance. Morale humanitaire, médias et politique, Paris, Gallimard,

2007 pour la présente édition (1993. Métailié); FAssin Didier, La Raison humanitaire. Une histoire morale du temps présent. Paris, Gallimard/Le Seuil, coll. « Hautes études », 2010
À travers ses agents ou les organisations syndicales des surveillants, l'Administration pénitentiaire alimente les médias ${ }^{31}$. Le ressenti de cette dernière continuera à s'exprimer pendant la guerre et sous Vichy. Des voix s'élèvent pour critiquer une campagne et des tentatives de réforme associées à l'esprit du Front populaire. Le tout est pris comme symbole de l'affaiblissement de tout principe d'autorité, de soumission à la populace parisienne au mépris des intérêts du pays alors que « le danger gronde à nos frontières ». La population est présentée comme victime de "sa majesté la presse » et de la subjectivité de certains journalistes (le juif et socialiste Alexis Danan est une cible de choix).

Plus encore, la contre-campagne accuse le mouvement d'avoir été contre-productif dans le champ même de la protection des mineurs. En 1941, Jean Bancal, docteur en droit, inspecteur général adjoint des services administratifs publie Essai sur le redressement de l'enfance coupable. En pleine période de Vichy, l'auteur juge que la campagne et les mesures gouvernementales n'ont servi qu'à " exciter les esprits des jeunes » et à distiller " un climat détestable dans les établissements ${ }^{32} »$.

Dans les années précédant la seconde guerre mondiale, des milieux qui ont porté la campagne se désengagent. Les mineurs de justice, plus coupables que victimes? Des porte-parole suspects? Le doute est bien là et d'autres causes intéressent l'opinion. Le choix des victimes est un arbitraire sur lequel les sociologues Luc Boltanski ou Didier Fassin s'interrogent dans leurs travaux respectifs ${ }^{33}$. Au sein même de la famille communiste, un déplacement est net après 1937. Le 
pouvoir de l'émotion embrasse d'autres figures de l'injustice: d'abord les orphelins ou réfugiés de la guerre d'Espagne. Dans un journal de la grande presse marchande comme Paris-Soir, la signature d'Alexis Danan est moins fréquente tant l'actualité internationale est prégnante. En 1938, le journaliste a pourtant gagné son procès contre Mettray, la justice a rendu hommage à l'homme et à sa corporation. L'écho est mince dans les colonnes de son propre journal. L'enfant de justice, éternelle cause éphémère? La campagne des années trente sera un instrument de l'application des réformes de 1945.

Dans l'opinion, dans les médias, l'enfant de justice est historiquement une cause particulièrement fragile. La société de l'information est néanmoins un cadre et un facteur dans l'individualisation du sujet qu'est le jeune, de sa transformation en victime et dans le développement d'un intérêt collectif pour la réforme des institutions. La campagne contre «les bagnes d'enfants " a sa place dans un cycle compassionnel qui ne s'inscrit que très partiellement dans le seul intérêt qu'inspire la question de la justice du mineur. L'efficacité est maximale lorsqu'elle est en capacité de capter des émotions plus larges: l'enfant, la patrie, la classe sociale. Le courant réformateur se développe dans une temporalité plus longue mais apprend à jouer avec une opinion versatile mais en état de peser sur le calendrier politique. Les années trente sont celles de l'entrée en force des médias et d'une société civile élargie dans le champ des politiques de l'enfant de justice. Avec la compassion et au prix de l'émotion, elles ont renforcé et enrichi des représentations à l'origine d'une culture sur le devoir du changement. Les voix sont variées. Déjà, les arguments trouvent dans la plasticité du langage des droits de l'enfant, le moyen de s'écouter. La légende noire des bagnes d'enfants est dans les mémoires lorsque les réformateurs de l'après 1945 obtiennent les moyens politiques, puis matériels et financiers d'institutionnaliser le secteur de la rééducation.

La campagne contre les «bagnes d'enfants » exprime un changement de forme et d'échelle dans les sensibilités socialement exprimées. Sujet et objet de mobilisations de différents groupes sociaux et d'institutions, l'enfant est investi moralement et politiquement. Il se fait "cause " dont les enjeux dépassent sa seule protection. L'action de l'entre-deux-guerres n'est pas neutre. À leur échelle et par ricochets sur un temps long, les processus en œuvre participent à une requalification des rapports sociaux et culturels entre les générations. 